\title{
$q$-Eulerian polynomials and polynomials with only real zeros *
}

\author{
Shi-Mei Ma and Yi Wang ${ }^{\dagger}$ \\ Department of Applied Mathematics \\ Dalian University of Technology \\ Dalian 116024, P. R. China \\ simons_ma@yahoo.com.cn \\ wangyi@dlut.edu.cn
}

Submitted: Jul 1, 2007; Accepted: Jan 4, 2008; Published: Jan 21, 2008

Mathematics Subject Classification: 05A15, 26C10

\begin{abstract}
Let $f$ and $F$ be two polynomials satisfying $F(x)=u(x) f(x)+v(x) f^{\prime}(x)$. We characterize the relation between the location and multiplicity of the real zeros of $f$ and $F$, which generalizes and unifies many known results, including the results of Brenti and Brändén about the $q$-Eulerian polynomials.
\end{abstract}

\section{Introduction}

Let $\mathscr{S}_{n}$ denote the symmetric group on the set $\{1,2, \ldots, n\}$ and $\pi=a_{1} a_{2} \cdots a_{n} \in \mathscr{S}_{n}$. An excedance in $\pi$ is an index $i$ such that $a_{i}>i$. Let exc $(\pi)$ denote the number of excedances in $\pi$. The classical Eulerian polynomials $A_{n}(x)$ are defined by

$$
A_{0}(x)=1, \quad A_{n}(x)=\sum_{\pi \in \mathscr{S}_{n}} x^{\operatorname{exc}(\pi)+1} \quad \text { for } n \geq 1,
$$

and have been extensively investigated. It is well known that the classical Eulerian polynomials satisfy the recurrence relation

$$
A_{n+1}(x)=(n+1) x A_{n}(x)+x(1-x) A_{n}^{\prime}(x)
$$

(see Bóna [1, p. 23] for instance). In [5], Brenti considered a $q$-analog of the classical Eulerian polynomials defined by

$$
A_{0}(x ; q)=1, \quad A_{n}(x ; q)=\sum_{\pi \in \mathscr{S}_{n}} x^{\operatorname{exc}(\pi)} q^{c(\pi)} \quad \text { for } n \geq 1
$$

*This work was supported by the National Science Foundation of China under grant number 10771027.

${ }^{\dagger}$ Corresponding author. 
where $c(\pi)$ is the number of cycles in $\pi$. The first few of the $q$-Eulerian polynomials are

$$
A_{0}(x ; q)=1, A_{1}(x ; q)=q, A_{2}(x ; q)=q(x+q), A_{3}(x ; q)=q\left[x^{2}+(3 q+1) x+q^{2}\right] .
$$

Clearly, $A_{n}(x)=x A_{n}(x ; 1)$ for $n \geq 1$. Brenti obtained the recurrence relation

$$
A_{n+1}(x ; q)=(n x+q) A_{n}(x ; q)+x(1-x) \frac{d}{d x} A_{n}(x ; q)
$$

([5, Proposition 7.2]) and showed that $A_{n}(x ; q)$ has only real nonpositive simple zeros when $q$ is a positive rational number ([5, Theorem 7.5]). He also proposed the following.

Conjecture 1 ([5, Conjecture 8.8]). Let $n, t \in \mathbb{N}$. Then $A_{n}(x ;-t)$ has only real zeros.

The conjecture has been settled recently by Brändén [3]. Let

$$
E_{n}(x ; q)=(1+x)^{n} A_{n}\left(\frac{x}{1+x} ; q\right) .
$$

Then it is clear that $A_{n}(x ; q)$ has only real zeros if and only if $E_{n}(x ; q)$ does. The recurrence (1) induces

$$
E_{n+1}(x ; q)=q(1+x) E_{n}(x ; q)+x(1+x) \frac{d}{d x} E_{n}(x ; q),
$$

with $E_{0}(x ; q)=1$. Using multiplier $n$-sequences, Brändén can prove that if $q>0, n+q \leq 0$ or $q \in \mathbb{Z}$, then $E_{n}(x ; q)$ has only real zeros, and so does $A_{n}(x ; q)$ (see [3, Theorem 6.3] for details). In the next section, we will obtain a more precise result directly by the recurrence (1) as an application of our main results in this paper.

Polynomials with only real zeros arise often in combinatorics, algebra, analysis, geometry, probability and statistics. For example, let $S(n, k)$ be the Stirling numbers of the second kind and $B_{n}(x)=\sum_{k=0}^{n} S(n, k) x^{k}$ the Bell polynomials. Then

$$
B_{n}(x)=x B_{n-1}(x)+x B_{n-1}^{\prime}(x), \quad B_{0}(x)=1 .
$$

For showing that the Stirling behavior is asymptotically normal, Harper [8] showed that the Bell polynomials have only real simple zeros by means of the recurrence (2).

Let RZ denote the set of real polynomials with only real zeros. Furthermore, denote by $\operatorname{RZ}(I)$ the set of such polynomials all of whose zeros are in the interval $I$. Suppose that $f, F \in$ RZ. Let $\left\{r_{i}\right\}$ and $\left\{s_{j}\right\}$ be all zeros of $f$ and $F$ in nonincreasing order respectively. We say that $f$ separates $F$, denoted by $f \preceq F$, if $\operatorname{deg} f \leq \operatorname{deg} F \leq \operatorname{deg} f+1$ and

$$
s_{1} \geq r_{1} \geq s_{2} \geq r_{2} \geq s_{3} \geq r_{3} \geq \cdots .
$$

It is well known that if $f \in \mathrm{RZ}$, then $f^{\prime} \in \mathrm{RZ}$ and $f^{\prime} \preceq f$. Following Wagner [13], a real polynomial is called standard if it has positive leading coefficient.

Let $f$ and $F$ be two polynomials satisfying the relation $F(x)=u(x) f(x)+v(x) f^{\prime}(x)$. A natural question is in which cases $f$ has only real zeros implies that $F$ does. There have been some partial results $[10,14]$. However, these results can not tell us the relation of the multiplicity and location of zeros of $f$ and $F$. The main object of this paper is to provide a characterization for such a problem, which can give a unified explanation of many known results. 


\section{Main results}

In this section we present the main results of this paper.

Theorem 2. Let $f$ and $F$ be two standard polynomials satisfying the relation

$$
F(x)=u(x) f(x)+v(x) f^{\prime}(x)
$$

where $u(x), v(x)$ are real polynomials and $\operatorname{deg} F=\operatorname{deg} f$ or $\operatorname{deg} f+1$. Assume that $f \in \mathrm{RZ}$ and $v(r) \leq 0$ whenever $f(r)=0$. Then $F \in \mathrm{RZ}$ and $f \preceq F$. Moreover, if $r$ is a zero of $f$ with the multiplicity $m$, then the multiplicity of $r$ as a zero of $F$ is

(a) $m-1$ if $v(r) \neq 0$; or

(b) $m$ if $v(r)=0$ but $u(r)+m v^{\prime}(r) \neq 0$; or

(c) $m+1$ if $v(r)=0$ and $u(r)+m v^{\prime}(r)=0$.

Furthermore, we have the following result.

(A) Suppose that $f \in \mathrm{RZ}(-\infty, r]$, where $r$ is the largest zero of $f$, with the multiplicity $m$. Then $F \in \mathrm{RZ}(-\infty, r]$ if and only if $v(r)=0$ and $u(r)+m v^{\prime}(r) \geq 0$.

(B) Suppose that $f \in \mathrm{RZ}[r,+\infty)$, where $r$ is the smallest zero of $f$, with the multiplicity $m$. Then $F \in \operatorname{RZ}[r,+\infty)$ if and only if $\operatorname{deg} F=\operatorname{deg} f$, or $v(r)=0$ and $u(r)+$ $m v^{\prime}(r) \leq 0$.

Proof. The first part of the statement about $F \in \mathrm{RZ}$ and $f \preceq F$ follows from [10, Theorem 2.1]. However, we give a direct proof of it for our purpose. Without loss of generality, we may assume that $f$ and $F$ are monic. Let $f(x)=\prod_{i=1}^{k}\left(x-r_{i}\right)^{m_{i}}$ where $r_{1}, \ldots, r_{k}$ are distinct zeros of $f(x)$ with the multiplicities $m_{1}, \ldots, m_{k}$ respectively. Then $\prod_{i=1}^{k}\left(x-r_{i}\right)^{m_{i}-1} \mid F(x)$. Denote $g(x)=\prod_{i=1}^{k}\left(x-r_{i}\right)$ and $G(x)=F(x) / \prod_{i=1}^{k}\left(x-r_{i}\right)^{m_{i}-1}$. Then $\operatorname{deg} G-\operatorname{deg} g=\operatorname{deg} F-\operatorname{deg} f=0$ or 1 , and by (3),

$$
G(x)=u(x) g(x)+v(x) \sum_{i=1}^{k} \frac{m_{i} g(x)}{x-r_{i}} .
$$

Consider first the case $v\left(r_{i}\right)<0$ for all $i$. Let $r_{k}<\cdots<r_{1}$. Then by (4), the sign of $G\left(r_{i}\right)$ is $(-1)^{i}$ for $i=1, \ldots, k$. Note that $G(x)$ is monic and $\operatorname{deg} G-\operatorname{deg} g=\operatorname{deg} F-\operatorname{deg} f$. Hence $G(x)$ has precisely one zero in each of $k$ intervals $\left(r_{k}, r_{k-1}\right), \ldots,\left(r_{2}, r_{1}\right),\left(r_{1},+\infty\right)$ and has an additional zero in the interval $\left(-\infty, r_{k}\right)$ if $\operatorname{deg} G-\operatorname{deg} g=1$. Thus $G \in \operatorname{RZ}$ and $g \preceq G$. It implies that $F \in \mathrm{RZ}$ and $f \preceq F$. Clearly, $r_{i}$ is not a zero of $G$. So $r_{i}$ is a zero of $F$ with the multiplicity $m_{i}-1$. This proves (a).

Next consider the general case. Let $v_{j}(x)=v(x)-1 / j$ and $F_{j}(x)=u(x) f(x)+$ $v_{j}(x) f^{\prime}(x)$. Then $v_{j}\left(r_{i}\right)<0$ for all $i$ when $j$ is sufficiently large, and so $F_{j} \in \mathrm{RZ}$ and $f \preceq F_{j}$. It is well known that the zeros of a polynomial are continuous functions of the coefficients of the polynomial and the limit of a sequence of RZ polynomials is still a RZ 
polynomial (see [7] for instance). Thus $F \in \mathrm{RZ}$ and $f \preceq F$ by continuity. Assume now that $v(r)=0$ for some zero $r$ of $f$ with the multiplicity $m$. Then $(x-r)^{m} \mid f$ implies $(x-r)^{m} \mid F$ from $(3)$. Let $f(x)=(x-r)^{m} h(x)$ and $F(x)=(x-r)^{m} H(x)$. Then $h(r) \neq 0$ and

$$
H(x)=\left[u(x)+m \frac{v(x)}{x-r}\right] h(x)+v(x) h^{\prime}(x)
$$

by (3). So $H(r)=\left[u(r)+m v^{\prime}(r)\right] h(r)$. If $u(r)+m v^{\prime}(r) \neq 0$, then $H(r) \neq 0$, and so the multiplicity of $r$ as a zero of $F$ is precisely $m$. This proves (b). If $u(r)+m v^{\prime}(r)=0$, then $H(r)=0$ and so the multiplicity of $r$ as a zero of $F$ is at least $m+1$. However, $f \preceq F$ and $r$ is a zero of $f$ with the multiplicity $m$. Hence the multiplicity of $r$ as a zero of $F$ is at most $m+1$. Thus the multiplicity of $r$ as a zero of $F$ is precisely $m+1$. This proves (c).

(A) Now let $r$ be the largest zero of $f$, with the multiplicity $m$. Then $F$ has at most one zero larger than $r$ since $f \preceq F$.

Assume that $v(r) \neq 0$. Then $r$ is a zero of $F$ with the multiplicity $m-1$. Thus $F$ has one zero larger than $r$. Assume that $v(r)=0$ and $u(r)+m v^{\prime}(r)<0$. Then $h(r)>0$ since $h$ is standard and has no zero larger than $r$. Hence $H(r)=\left[u(r)+m v^{\prime}(r)\right] h(r)<0$. Thus $H$ has one zero larger than $r$ since $H$ is standard, and so does $F$. Assume that $v(r)=0$ and $u(r)+m v^{\prime}(r)>0$. Then $H(r)>0$. Hence $H$ has an even number of zeros larger than $r$. Thus $H$ has no zero larger than $r$, and so does $F$. Assume that $v(r)=u(r)+m v^{\prime}(r)=0$. Then $r$ is a zero of $F$ with the multiplicity $m+1$. Thus $F$ has no zero larger than $r$.

So we conclude that $F \in \mathrm{RZ}(-\infty, r]$ if and only if $v(r)=0$ and $u(r)+m v^{\prime}(r) \geq 0$.

(B) If $\operatorname{deg} F=\operatorname{deg} f$, then the result is clear since $f \preceq F$. If $\operatorname{deg} F=\operatorname{deg} f+1$, then let $g(x)=(-1)^{n} f(-x)$ and $G(x)=(-1)^{n+1} F(-x)$ where $n=\operatorname{deg} f$. It follows that

$$
G(x)=-u(-x) g(x)+v(-x) g^{\prime}(x)
$$

from (3). Thus the statement follows from (A).

Combining (A) and (B) of Theorem 2, it is not difficult to give a necessary and sufficient condition that guarantees zeros of $f$ and $F$ are in the same closed interval. We omit the details for the sake of brevity and only give the following result as a demonstration. As usual, let $x^{m} \| f(x)$ denote $x^{m} \mid f(x)$ but $x^{m+1} \nmid f(x)$.

Corollary 3. Let $f$ and $F$ be two standard polynomials satisfying

$$
F(x)=(a x+b) f(x)+x(x+1) f^{\prime}(x) .
$$

Suppose that $f(x) \in \mathrm{RZ}[-1,0], x^{m_{0}} \| f$ and $(x+1)^{m_{1}} \| f$. Then $b+m_{0} \geq 0$ and $a+m_{1} \geq b$ imply that $F \in \mathrm{RZ}[-1,0]$ and $f \preceq F$. Furthermore, $x^{m_{0}} \| F$ if $b+m_{0}>0$ or $x^{m_{0}+1} \| F$ if $b+m_{0}=0$, and $(x+1)^{m_{1}} \| F$ if $a+m_{1}>b$ or $(x+1)^{m_{1}+1} \| F$ if $a+m_{1}=b$.

Now we can apply Theorem 2 to strengthen the results of Brenti and Brändén about the $q$-Eulerian polynomials by the recurrence (1) and by induction. 
When $q=-t$ is a negative integer, (5) can be written as

$$
A_{n}(x ;-t)=\sum_{k=0}^{n}(-1)^{k}\left(\begin{array}{l}
t \\
k
\end{array}\right) k ! S(n, k)(x-1)^{n-k} .
$$

It immediately follows that $(x-1)^{n-t} \| A_{n}(x ;-t)$ for $n \geq t$, as desired.

\section{Applications}

Theorem 2 can provide a unified explanation of many known results, including the fact that the classical Eulerian polynomials and the Bell polynomials have only real simple zeros. In this section we give more examples as applications.

\subsection{Linear transformations preserving RZness}

Consider the invertible linear operator $\mathcal{T}: \mathbb{R}[x] \rightarrow \mathbb{R}[x]$ defined by

$$
\mathcal{T}\left((x)_{i}\right)=x^{i}
$$

for all $i \in \mathbb{N}$ and linear extension, where $(x)_{i}=x(x-1) \cdots(x-i+1)$ and $(x)_{0}=1$. Wagner [13, Lemma 3.3] showed the following result.

Proposition 6. Let $\xi \in \mathbb{R}$ and let $p$ be a real polynomial such that $\mathcal{T}(p) \in \mathrm{RZ}(-\infty, 0]$. Then

(a) $F:=\mathcal{T}((x-\xi) p) \in \mathrm{RZ}$.

(b) Let $m$ denote the multiplicity of 0 as a zero of $\mathcal{T}(p)$. Then $F \in \mathrm{RZ}(-\infty, 0]$ if and only if $\xi \leq m$.

(c) Furthermore, the multiplicity of 0 as a zero of $F$ is $m$ if $\xi \neq m$, and is at least $m+1$ if $\xi=m$.

Actually, let $f=\mathcal{T}(p)$. Then $F=(x-\xi) f+x f^{\prime}$. Thus Proposition 6 is obvious from the viewpoint of Theorem 2 .

The E-transformation is the invertible linear operator $\mathcal{E}: \mathbb{R}[x] \rightarrow \mathbb{R}[x]$ defined by

$$
\mathcal{E}\left(\left(\begin{array}{l}
x \\
i
\end{array}\right)\right)=x^{i}
$$

for all $i \in \mathbb{N}$ and linear extension. This transformation is important in the theory of $(P, \Omega)$ partitions (see Brenti [4] for details). Brändén [3, Lemma 4.4] showed the following.

Proposition 7. Let $\alpha \in[-1,0]$ and let $p$ be a polynomial such that $\mathcal{E}(p) \in \mathrm{RZ}[-1,0]$. Then $\mathcal{E}((x-\alpha) p) \in \mathrm{RZ}[-1,0]$ and $\mathcal{E}(p) \preceq \mathcal{E}((x-\alpha) p)$. If $\mathcal{E}(p)$ in addition only has simple zeros, then so does $\mathcal{E}((x-\alpha) p)$. 
Actually, let $f=\mathcal{E}(p)$ and $F=\mathcal{E}((x-\alpha) p)$. Then $F=(x-\alpha) f+x(x+1) f^{\prime}$. Thus Proposition 7 is an immediate consequence of Corollary 3. Furthermore, if the multiplicity of 0 as a zero of $f$ is $m_{0}$, then the multiplicity of 0 as a zero of $F$ is also $m_{0}$ except $m_{0}=\alpha=0$; if the multiplicity of -1 as a zero of $f$ is $m_{1}$, then the multiplicity of -1 as a zero of $F$ is also $m_{1}$ except $m_{1}=0$ and $\alpha=-1$.

\subsection{Compositions of multisets}

Let $\mathbf{n}=\left(n_{1}, n_{2}, \ldots\right)$ be the multiset consisting of $n_{i}$ copies of the $i$ th type element. A composition of $\mathbf{n}$ is an expression of $\mathbf{n}$ as an ordered partition of nonempty multisets. Denote by $\mathcal{O}(\mathbf{n}, k)$ the number of compositions of $\mathbf{n}$ into exactly $k$ parts. Then

$$
\left(n_{j}+1\right) \mathcal{O}\left(\mathbf{n}+e_{j}, k\right)=k \mathcal{O}(\mathbf{n}, k-1)+\left(n_{j}+k\right) \mathcal{O}(\mathbf{n}, k),
$$

where $\mathbf{n}+e_{j}$ denotes the multiset obtained from $\mathbf{n}$ by adjoining one (additional) copy of the $j$ th type element. Let $f_{\mathbf{n}}(x)=\sum_{k \geq 0} \mathcal{O}(\mathbf{n}, k) x^{k}$ be the associated generating function. Then by (6),

$$
\left(n_{j}+1\right) f_{\mathbf{n}+e_{j}}(x)=\left(x+n_{j}\right) f_{\mathbf{n}}(x)+x(x+1) f_{\mathbf{n}}^{\prime}(x) .
$$

Simion showed that the multiplicity of -1 as a zero of $f(x)$ is $\max _{i}\left\{n_{i}-1\right\}$ by means of the theory of posets ([11, Lemma 1.1]). Based on this result and appropriate transformation to the recurrence (7), she further showed that $f_{\mathbf{n}}(x) \in \mathrm{RZ}[-1,0]$ and $f_{\mathbf{n}}(x) \preceq f_{\mathbf{n}+e_{j}}(x)$ ([11, Theorem 1]). These results are now clear from the viewpoint of Corollary 3 .

In particular, if $\mathbf{n}=(1,1, \ldots, 1)$, then $\mathcal{O}(\mathbf{n}, k)=k ! S(n, k)$, where $S(n, k)$ is the Stirling number of the second kind. Thus the polynomial $F_{n}(x)=\sum_{k=1}^{n} k ! S(n, k) x^{k}$ has only real simple zeros in the interval $[-1,0]$. It is interesting that $F_{n}(x)=\frac{x^{n+1}}{x+1} A_{n}\left(\frac{x+1}{x}\right)$ by the Frobenius formula, where $A_{n}(x)$ is the classical Eulerian polynomial.

\subsection{Alternating runs}

Let $\pi=a_{1} a_{2} \cdots a_{n} \in \mathscr{S}_{n}$. We say that $\pi$ changes direction at position $i$ if either $a_{i-1}<$ $a_{i}>a_{i+1}$, or $a_{i-1}>a_{i}<a_{i+1}$. We say that $\pi$ has $k$ alternating runs if there are $k-1$ indices $i$ such that $\pi$ changes direction at these positions. Let $R(n, k)$ denote the number of permutations in $\mathscr{S}_{n}$ having $k$ alternating runs. Then

$$
R(n, k)=k R(n-1, k)+2 R(n-1, k-1)+(n-k) R(n-1, k-2)
$$

for $n, k \geq 1$, where $R(1,0)=1$ and $R(1, k)=0$ for $k \geq 1$ (see Bóna [1, Lemma 1.37] for a combinatorial proof). Let $R_{n}(x)=\sum_{k=1}^{n-1} R(n, k) x^{k}$. Then the recurrence (8) induces

$$
R_{n+2}(x)=x(n x+2) R_{n+1}(x)+x\left(1-x^{2}\right) R_{n+1}^{\prime}(x),
$$

with $R_{1}(x)=1$ and $R_{2}(x)=2 x$. Bóna and Ehrenborg [2, Lemma 2.3] showed that $R_{n}(x)$ has the zero $x=-1$ with multiplicity $\left\lfloor\frac{n}{2}\right\rfloor-1$ and suspected that the other half zeros of 
$R_{n}(x)$ are all real, negative and distinct. The polynomial $R_{n}(x)$ is closely related to the classical Eulerian polynomial $A_{n}(x)$ :

$$
R_{n}(x)=\left(\frac{1+x}{2}\right)^{n-1}(1+w)^{n+1} A_{n}\left(\frac{1-w}{1+w}\right), \quad w=\sqrt{\frac{1-x}{1+x}}
$$

(Knuth [9, p. 605]). From the relation (10) and the fact that $A_{n}(x)$ has only real zeros, Wilf can show that $R_{n}(x)$ has only real zeros for $n \geq 2$ (see Bóna [1, Theorem 1.41] and Stanley [12] for details). Very recently, Canfield and Wilf [6] pointed out (without proof) that this result can also be obtained based on the recurrence (9). Indeed, we can give the following more precise result by Theorem 2 .

Corollary 8. Let $R_{n}(x)$ be the generating function of alternating runs. Then $R_{n}(x) \in$ $\mathrm{RZ}[-1,0]$ and $R_{n}(x) \preceq R_{n+1}(x)$ for $n \geq 1$. More precisely, $R_{n}(x)$ has $\left\lceil\frac{n}{2}\right\rceil$ simple zeros including $x=0$, and the zero $x=-1$ with the multiplicity $\left\lfloor\frac{n}{2}\right\rfloor-1$.

\section{Acknowledgments}

The authors thank the anonymous referee for careful reading and helpful suggestions.

\section{References}

[1] M. Bóna, Combinatorics of permutations, Chapman \& Hall/CRC, Boca Raton, FL, 2004.

[2] M. Bóna and R. Ehrenborg, A combinatorial proof of the log-concavity of the numbers of permutations with $k$ runs, J. Combin. Theory Ser. A 90 (2000) 293-303.

[3] P. Brändén, On linear transformations preserving the Pólya frequency property, Trans. Amer. Math. Soc. 358 (2006) 3697-3716.

[4] F. Brenti, Unimodal, log-concave, and Pólya frequency sequences in combinatorics, Mem. Amer. Math. Soc. 413 (1989).

[5] F. Brenti, A class of $q$-symmetric functions arising from plethysm, J. Combin. Theory Ser. A 91 (2000) 137-170.

[6] E. R. Canfield and H. Wilf, Counting permutations by their alternating runs, J. Combin. Theory Ser. A 115 (2008) 213-225.

[7] J. L. Coolidge, The continuity of the roots of an algebraic equation, Ann. of Math. (2) 9 (1908) 116-118.

[8] L. H. Harper, Stirling behavior is asymptotically normal, Ann. Math. Stat. 38 (1967) 401-414.

[9] D. E. Knuth, The Art of Computer Programming, vol. 3, Fundamental Algorithms, Addison-Wesley, Reading, MA, 1973. 
[10] L. Liu and Y. Wang, A unified approach to polynomial sequences with only real zeros, Adv. in Appl. Math. 38 (2007) 542-560.

[11] R. Simion, A multiindexed Sturm sequence of polynomials and unimodality of certain combinatorial sequences, J. Combin. Theory Ser. A 36 (1984) 15-22.

[12] R. P. Stanley, Longest alternating subsequences of permutations, math.CD/0511419.

[13] D. G. Wagner, The partition polynomials of a finite set system, J. Combin. Theory Ser. A 56 (1991) 138-159.

[14] Y. Wang and Y. -N. Yeh, Polynomials with real zeros and Pólya frequency sequences, J. Combin. Theory Ser. A 109 (2005) 63-74. 